NOVA

University of Newcastle Research Online

nova.newcastle.edu.au

Kouretzis, George P.; Krabbenhøft, Kristian; Sheng, Daichao; Sloan, Scott W. "Soil-buried pipeline interaction for vertical downwards relative offset" Canadian Geotechnical Journal Vol. 51, p. 1087-1094 (2014)

Available from: $\underline{\text { http://dx.doi.org/10.1139/cgj-2014-0029 }}$

Accessed from: $\underline{\text { http://hdl.handle.net/1959.13/1056711 }}$ 


\title{
Soil-buried pipeline interaction for vertical downwards relative offset
}

\author{
George P. Kouretzis ${ }^{1}$, Kristian Krabbenhøft, Daichao Sheng and Scott W. Sloan \\ ARC Centre of Excellence for Geotechnical Science and Engineering, Faculty of Engineering \\ and Built Environment, The University of Newcastle, Callaghan, NSW 2308, Australia
}

A new perspective is presented on the interaction effects for the vertical downwards offset of a pipeline relative to its surrounding soil. Instead of estimating the interaction force via shallow footing bearing capacity theory, as per common pipeline design practice, we assume that vertical movement of the pipeline in uniform soil is governed by mechanisms similar to the lateral loading of a circular pile up to its limit load. The validity of this assumption is investigated numerically with the finite element limit analysis method, and design expressions are derived for the maximum interaction force on pipelines embedded in cohesive and granular soils. For the common case of buried pipelines built in sand-backfilled trenches, the same numerical method is employed to determine the necessary trench dimensions so as to avoid interaction with the possibly much stiffer native soil, that will result in a significant increase of the force applied on the pipeline during ground movement. The described approach can be employed in project-specific analyses to optimize trench dimensions, and thus avoid unnecessary excavation costs or mitigation measures.

Keywords: buried pipelines; vertical offset; trench; design; limit analysis

\section{Introduction}

Permanent ground deformations of a magnitude sufficient to jeopardize the integrity of buried pipelines may take place in areas where their route crosses soil deposits that undergo significant volume changes due to environmental effects such as wetting-drying and freezing-thawing cycles (Rajeev \& Kodikara 2011, Oswell 2011); where they occur in irrigated lands where aquifer overpumping may result in nonuniform consolidation (Wols and van Thienen, 2014); where they are subject to surface settlement associated with mine subsidence or near-surface tunnelling works (Wang et al. 2011); where they span normal or oblique seismic fault crossings (Rojhani et al. 2012); where they are embedded in potentially unstable slopes (Cocchetti et al. 2009); or where they undergo liquefaction-induced lateral spreading (O' Rourke and Liu, 2012). Regardless of whether the ground surface deformation patterns are widespread or localized, the design of buried pipelines subjected to permanent ground movement is commonly based on numerical or analytical beamnonlinear spring models (e.g. ASCE-ALA, 2005; Karamitros et al., 2007, Karamitros et al. 2011). Input parameters of such models include the force-displacement curve of the elastoplastic soil springs which represent the surrounding native soil, or the backfill for cases of pipelines constructed in trenches. Numerous studies in the literature have focused on the development of force-displacement curves for horizontal offset (Trautmann and O' Rourke 1985, Olson 2009, Jung et al. 2013a, Yimsiri et al. 2004, di Prisco and Galli 2006, Kouretzis et al. 2013, Robert and Soga 2010, Turner 2004, Paulin et al. 1998) and vertical upwards offset (Trautmann et. al, 1985, Yimsiri et al. 2004, Jung et al. 2013b). Much less attention has been paid to

\footnotetext{
${ }^{1}$ Corresponding author - email: Georgios.Kouretzis@newcastle.edu.au tel. +61 24921 6449, postal address: EA Building, University of Newcastle, Callaghan NSW 2308, Australia
} 
the problem of vertical downwards offset, although the corresponding maximum force applied on the pipeline can prove critical for its design. For that case, established design guidelines (ASCE-ALA, 2005) and state-of-practice references (O' Rourke and Liu, 2012) suggest that the problem resembles to that of the vertical loading of a shallow strip footing, and propose the use of the common bearing capacity equations to estimate the maximum force on the pipeline per unit length:

(1) $Q_{d}=N_{c} c D+N_{q} \gamma^{\prime} H D+N_{\gamma} \gamma(D / 2)^{2}$

where $D$ is the pipeline diameter, $H$ is the depth to the pipeline centerline, $V$ is the total and $\gamma^{\prime}$ is the effective unit weight of the soil, $c$ is the cohesion and $N_{c}, N_{q}, N_{y}$ are the common bearing capacity factors that can be estimated as (ASCE-ALA, 2005):

$$
N_{c}=\cot (\varphi+0.001) \cdot\left\{e^{\pi \tan (\varphi+0.001)} \cdot \tan ^{2}\left(45+\frac{\varphi+0.001}{2}\right)-1\right\}
$$

$$
N_{q}=e^{\pi \tan \varphi} \cdot \tan ^{2}\left(45+\frac{\varphi}{2}\right)
$$

(4) $N_{\gamma}=e^{(0.18 \varphi-2.5)}$

with $\varphi$ being the soil friction angle. The rationale behind employing the bearing capacity equations for a shallow strip footing to estimate the maximum force on a buried pipeline during vertical downwards movement is quite straightforward: as the embedment depth of the pipeline $H$ tends to zero, the pipeline will essentially behave as a shallow strip footing of width $D$ (Figure 1a). However, as the pipeline embedment increases, the failure mode will change to a "deep" one, and the maximum force developing on the pipeline will approach the ultimate horizontal load of a laterally loaded circular pile in uniform ground (Figure 1b).

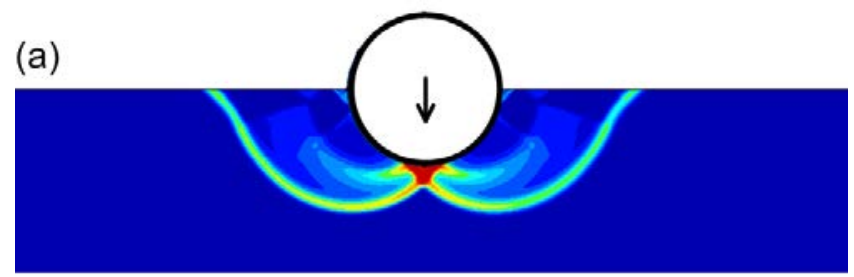

(b)

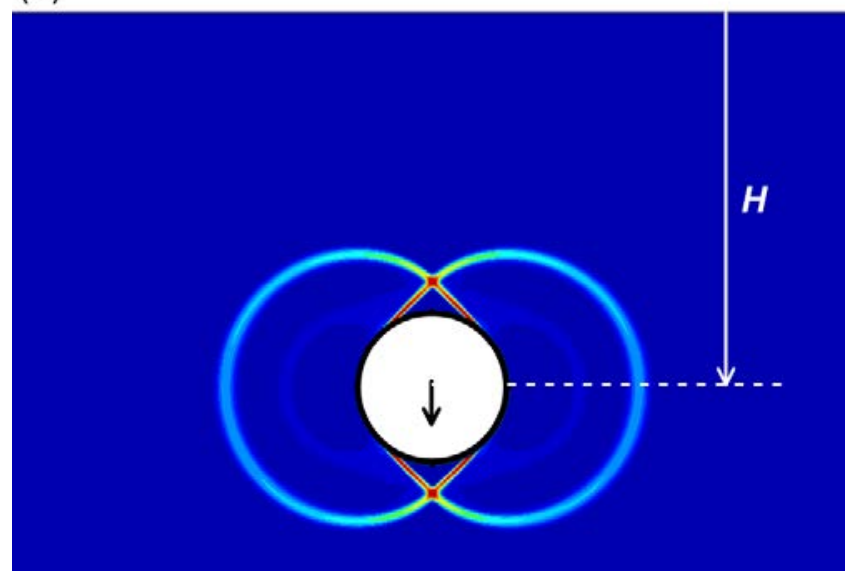

Fig. 1. Shear strain contours defining the failure surface around a vertically loaded (a) pipeline resting on the surface $(H / D=0)$ of a uniform cohesive soil layer, and (b) the same pipeline embedded at depth $H / D=2.5$, sufficient for the development of a "deep" failure mode. The presented failure surfaces correspond to undrained loading conditions. 
The scope of this paper is (i) to investigate numerically the effect of considering the actual pipeline embedment conditions on the maximum force developed during vertical downwards movement, (ii) to compare the results with the bearing capacity eq. (1), and (iii) to propose an updated method for estimating the vertical elastoplastic spring properties to be used as input in beam-nonlinear spring models. The finite element limit analysis method which forms the basis of this study is briefly presented in the first part, together with the results of benchmark analyses employed for its validation against experimental, analytical and numerical results from the literature. Accordingly, the maximum force on the pipeline is estimated parametrically for pipelines embedded in cohesive and granular soils, as a function of the problem geometry and soil characteristics. Finally, a discussion is given for the ordinary case of pipelines constructed in sand-backfilled trenches, where interaction with the stiff native soil can result in uncontrolled increase of the force applied on the pipeline. Practical guidelines are provided to dimension the trench so as to avoid such effects.

\section{Mechanisms of the downwards relative offset problem and validation of the numerical methodology}

The focus of this study is to estimate the maximum force on the pipeline, to be used for the definition of the elastoplastic soil springs, rather than the complete forcedisplacement curve during vertical differential offset between the pipeline and the soil. The yield displacement of the springs can be assumed to be of the order of 0.10D to 0.15D for granular and cohesive soils (ASCE-ALA, 2005, O' Rourke and Liu, 2012). The study employs the finite element limit analysis method developed at Newcastle University by Sloan $(1988,1989)$, Sloan \& Kleeman (1995), Lyamin \& Sloan (2002a, 2002b), Krabbenhøft et al. (2005, 2007) and Sloan (2013). The code is under commercial development by Optum Computational Engineering (2014), and computes accurate estimates of the collapse load very efficiently. This method has been applied successfully to a wide variety of geotechnical problems (Sloan, 2013), including the pull-out strength of anchors and the penetration resistance of offshore pipelines (Merifield et al., 2001, Merifield and Sloan, 2006, Martin and White, 2012). In the following sections we present a number of benchmark analyses to verify the adopted numerical methodology against analytical solutions based on classical plasticity theory and experimental/numerical results from the literature, as well as to investigate the mechanisms governing the problem of vertical downwards relative offset.

\subsection{Pipeline in cohesive soil}

As discussed in the introduction, we assume here that when the pipeline embedment depth is sufficient, a "deep" failure mode will develop (Figure 1b), resembling that of a circular pile subjected to its ultimate horizontal load. The exact ultimate horizontal load $Q_{d}$ (per unit length) of a circular pile in weightless cohesive soil was calculated by Randolph and Houlsby (1984) as:

$$
\begin{aligned}
& Q_{d} / S_{u} D=9.14 \quad \text { for a "smooth" pile } \\
& Q_{d} / S_{u} D=11.94 \quad \text { for a "rough" pile }
\end{aligned}
$$

where $S_{u}$ is the undrained shear strength of the soil. On the other hand, in the special case of a shallow strip footing on weightless Tresca soil, eq. (1) reduces to the exact solution $Q_{d} / S_{u} D=5.14$, known from plasticity theory. In order to verify the accuracy of the numerical methodology against these two extreme cases, and to establish the transition embedment ratio $H / D$ where the behaviour changes from that of a strip footing to that of a long laterally-loaded pile, a series of parametric analyses were performed with the embedment ratio ranging between $H / D=0$ and $H / D=6$. 


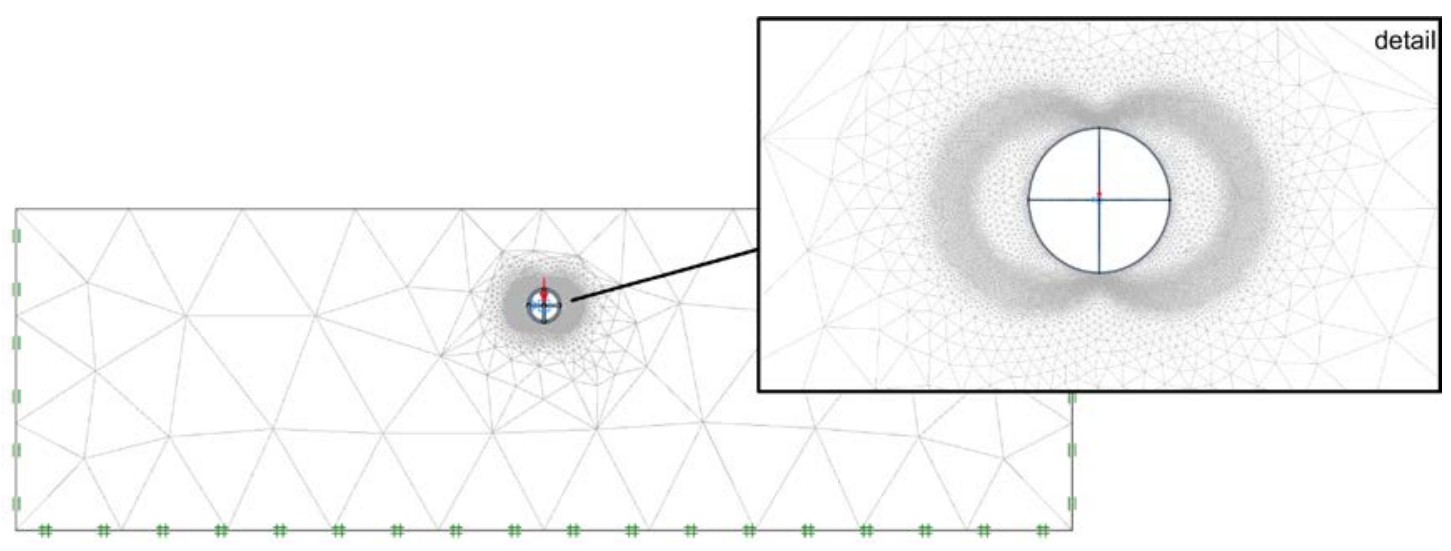

Fig. 2. Finite element limit analysis mesh used for the simulation of pipeline vertical relative offset into cohesive soil, under undrained conditions. The presented mesh corresponds to pipeline embedment ratio $H / D=3$.

The pipeline is assumed to be rigid, in line with previous similar studies (Yimsiri et al. 2004, Kouretzis et al. 2013, Jung et al. 2013a, Jung et al. 2013b). This however does not suggest that the results are not valid for relatively flexible pipelines too, provided that the deformation of the pipeline section is small compared to its dimensions, and infinitesimal strain theory applies. Both "smooth" and "rough" pipelines are considered; i.e. we assume a simple Coulomb friction law for the soil-pipeline interface, with the strength reduction factor ranging from $\delta=0$ for the "smooth" pipeline to $\delta=1$ for the "rough" pipeline. No tensile stresses are allowed to develop at the interface via introducing a tension cut-off. All the results presented in this section, and in this study in general, correspond to the upper bound solution. Preliminary analyses revealed that, as predicted by limit analysis theory (Chen and Han, 2007), when a sufficient number of elements are employed in the analyses (of the order of 50,000 -Figure 2), the difference between the upper bound and the lower bound collapse loads is less than 1-2\%. Employing mesh adaptivity based on a shear dissipation criterion (Optum Computational Engineering, 2014) leads to the automatic refinement of the mesh at the area of interest, where the failure surface develops (Figure 2), and to an accurate solution at minimal computational cost.

Results of the parametric analyses for cohesive weightless soil are presented in Figure 3 , illustrating the variation of the maximum normalized force $Q_{d} / S_{u} D$ developing on the pipeline (which is the collapse load) with various embedment ratios $H / D$. Two major conclusions are drawn:

- The numerical results approach the exact analytical solutions for the two extreme cases of a shallow strip footing $(H / D=0)$ and of a circular pile in uniform ground $(H / D>2)$. A divergence is noticed at $H / D=0$, due to the curved shape of the pipeline compared to a flat strip footing.

- Transition from the "shallow" to the "deep" failure mode takes place at a relatively low value of the embedment ratio, of the order of $H / D=2$. Thus, adopting the bearing capacity eq. (1) for the estimation of the vertical force will result in a substantial underestimation of the maximum force for many buried pipelines. Note that, unlike the bearing capacity problem of a shallow footing, where underestimating the sustainable force will result in a more conservative design, in the displacement-controlled problem of a pipeline subjected to differential ground movement, underpredicting the resisting force will lead to underprediction of the pipeline strains. 


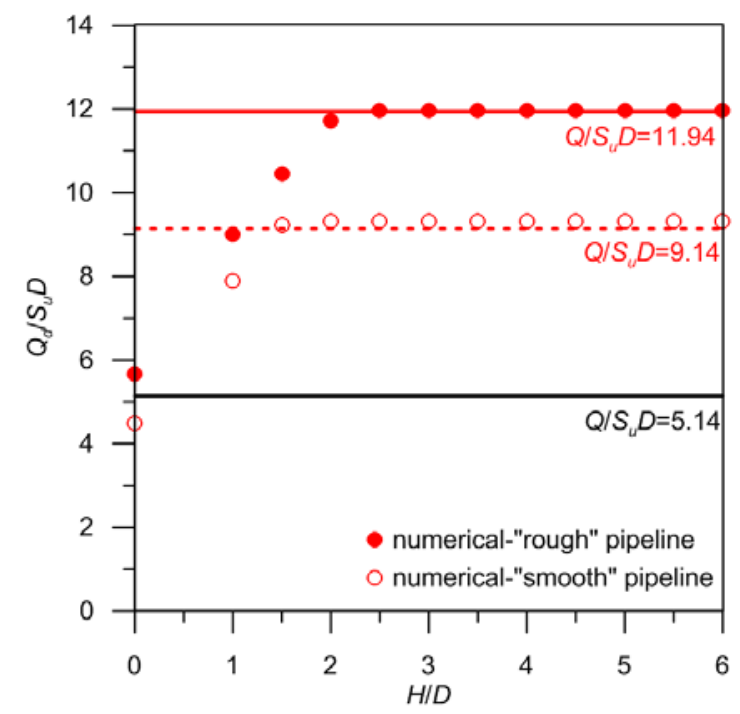

Fig. 3. Comparison of the exact solutions for a strip footing (eq. 1) and for a horizontally loaded circular pile in uniform weightless cohesive soil (eq. 5-6) with the results of finite element limit analyses, for increasing embedment ratio $H / D$.

\subsection{Pipeline in granular soil}

In the absence of exact solutions or specific experimental data for vertical downwards pipeline offset in granular soils, we will validate the method on the experimental data by Trautmann et al. (1985) for vertical upwards movement, and the corresponding numerical results by Yimsiri et al. (2004). Trautmann et al. (1985) performed pull-out tests to estimate the maximum uplift force on a $D=0.104 \mathrm{~m}$ pipeline embedded in dry Cornell filter sand, at depths $H / D=1.5, H / D=4, H / D=8$ and $H / D=13$ (Figure 4a). Later, Yimsiri et al. (2004) succeeded in numerically replicating these tests for medium and dense sand with the displacement finite element method (Figure 5), using the classical Mohr-Coulomb and the NorSand models (Jefferies, 1993). Trautmann et al. (1985) report that the medium sand $\left(D_{r}=45 \%\right)$ had a unit weight of $\gamma=16.4 \mathrm{kN} / \mathrm{m}^{3}$ and the dense sand $\left(D_{r}=80 \%\right)$ a unit weight of $\gamma=17.7 \mathrm{~N} / \mathrm{m}^{3}$. Their corresponding peak friction angles, measured from direct shear tests under low normal stresses (which are compatible with the stress level during the uplift tests), were $\varphi_{\text {peak }}=36^{\circ}$ for the medium sand and $\varphi_{\text {peak }}=44^{\circ}$ for the dense sand. The critical state friction angle determined during the direct shear tests was $\varphi_{\text {crit }}=31^{\circ}$. Furthermore, Yimsiri et al. (2004) estimated the dilation angle $\psi$ of the medium and dense sand on the basis of the expression $\varphi_{\text {peak }}=\varphi_{\text {crit }}+0.8 \psi$ (Bolton, 1986), giving $\psi=6^{\circ}$ for the medium sand and $\psi=16^{\circ}$ for the dense sand. In order to simulate the laboratory and numerical pipeline uplift experiments with the finite element limit analysis method, we will follow the approach proposed by Davis (1968) for materials with non-associative coaxial flow rule i.e. we will assume that the sand follows the Mohr-Coulomb failure criterion, and replace the peak friction angle $\varphi$ with an equivalent angle $\varphi^{*}$, assuming an associative flow rule. This equivalent angle $\varphi^{*}$ is given by (Davis, 1968):

(7) $\tan \varphi^{*}=n \tan \varphi$

where

(8)

$\cos \psi \cos \varphi$

$\mathrm{n}=\frac{\cos \psi \cos \varphi}{1-\sin \psi \sin \varphi}$ 

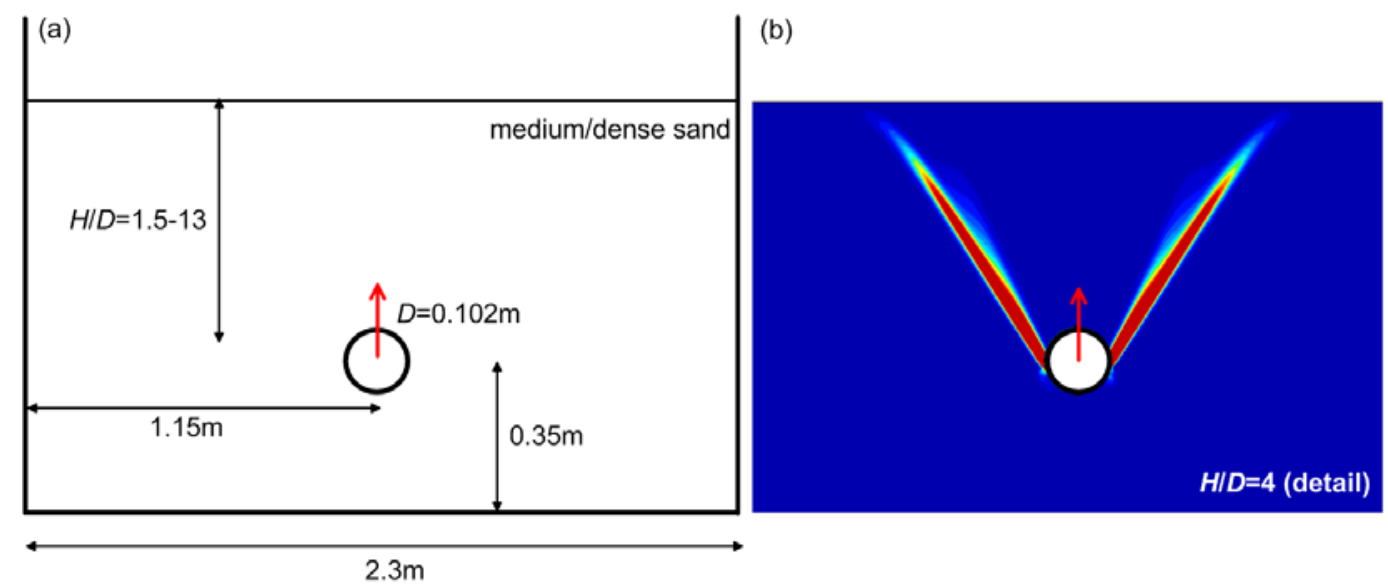

Fig. 4. (a) Schematic representation of the Trautmann et al. (1985) pull-out tests and (b) Failure mode during pipeline uplift from the benchmark analyses performed to replicate the experiments.

In other words, instead of considering e.g. $\varphi_{\text {peak }}=44^{\circ}$ and $\psi=16^{\circ}$ to simulate dense sand response during the Trautmann et al. experiments, we will assume associative flow and a sand friction angle of $\varphi^{*}=39.6^{\circ}$. Finite element limit analyses based on this concept were performed for medium and dense sand, and for a number of different embedment ratios $H / D$, in order to compare the resulting normalized uplift force $Q_{U} / Y H D$ - embedment ratio $H / D$ curves with the relevant data of Trautmann et al. (1985) and Yimsiri et al. (2004). The results are shown in Figure 5. In these analyses, the Coulomb interface friction angle $\delta$ between the pipeline and the sand was assumed to be $\delta=0.5 \varphi^{*}$, so as to be compatible with the values adopted by Yimsiri et al. (2004).

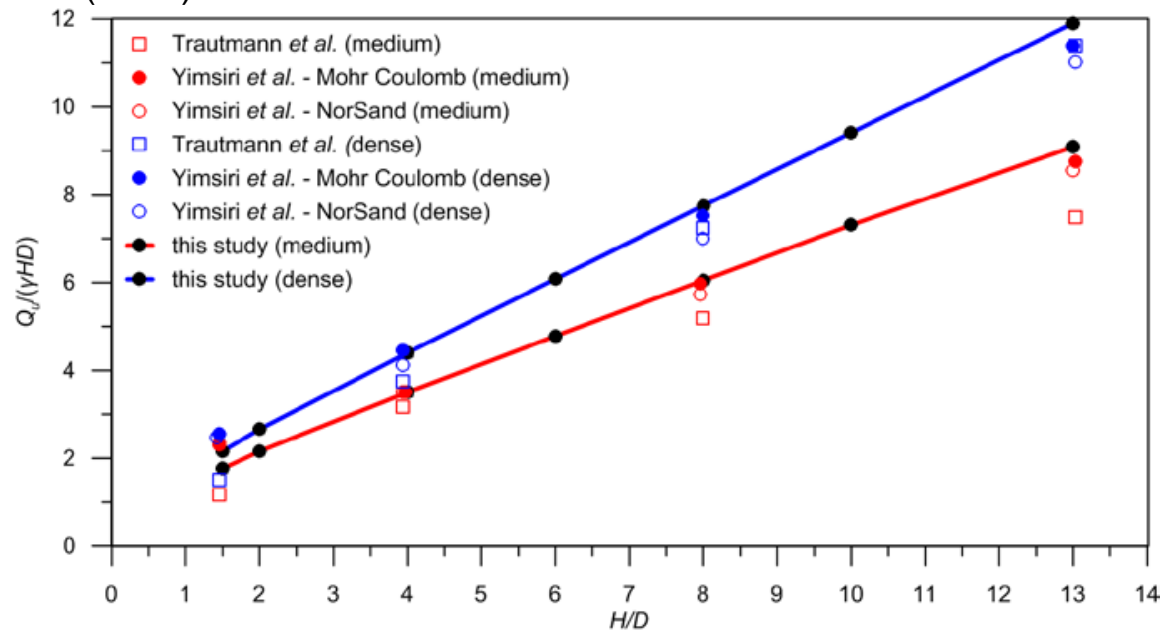

Fig. 5. Comparison of the experimentally measured normalized uplift force in medium and dense sand against displacement finite element (Yimsiri et al. 2004) and limit analysis (this study) numerical results.

Figure 5 indicates that the finite element limit analyses with the Davis shear strength parameters (eqs. 7-8) are successful in predicting the maximum force on the pipeline during the uplift experiments, both in medium and dense sand. The predictions are very similar to the results of the displacement finite element analyses obtained by Yimsiri et al. (2004) with the non-associative Mohr-Coulomb model, but also with the more complex critical state NorSand model. It can be argued though that finite element limit analysis tends to somewhat overestimate the peak force on the pipeline (Figure 5). This could be attributed to the fact that the numerical method does not account for large deformation effects, and the beneficial effect of separation at the 
soil-pipeline interface is not considered. Nevertheless, this will result in rather conservative predictions of pipeline strains. We can therefore employ the same concept for the simulation of soil-pipeline interaction effects during vertical downwards offset, as described later in section 4.

\section{Maximum bearing force for pipelines in cohesive soil}

As discussed in the previous Section 2.1, a better approximation of the maximum force developing on "deep" pipelines (with $H / D>2$ ) during vertical relative offset can be obtained by assuming that the problem is similar to a laterally-loaded circular pile. The exact solution by Randolph and Houlsby (1984) for the pile problem was derived while assuming that soil is weightless. Although this simplifying assumption will not introduce a significant error in the solution, at least for pipelines driven through clay with realistic undrained shear strengths, we can incorporate the effect of soil weight in the solution by adding the replaced soil weight in eq. (5-6) as:

(9) $Q_{d}=9.14 S_{u} D+\gamma \cdot \pi(D / 2)^{2} \quad$ for a "smooth" pipeline

(10) $Q_{d}=11.94 S_{u} D+\gamma \cdot \pi(D / 2)^{2} \quad$ for a "rough" pipeline

Figure 6a compares the predictions of eq. (9-10) against the numerical results for the rather extreme case of a $1 \mathrm{~m}$-diameter pipeline embedded in homogeneous undrained clay with $S_{u}=1 \mathrm{kPa}$ and $\gamma=15 \mathrm{kN} / \mathrm{m}^{3}$. Note that the reason for selecting such a low undrained shear strength is to underline the effect of the soil unit weight on the force exerted on the pipeline. A different scenario is examined in Figure $6 \mathrm{~b}$, with the pipeline being embedded in homogeneous very stiff soil with $S_{u}=100 \mathrm{kPa}$ and $\gamma=15 \mathrm{kN} / \mathrm{m}^{3}$, where the effect of soil weight becomes trivial. In Figure 6 we also include for comparison purposes the predictions of the bearing capacity eq. (1), as well as those from eq. (5-6) for weightless soil. Three sets of analyses are presented for each case: one assuming a perfectly "smooth" pipeline $(\delta=0)$, one a perfectly "rough" pipeline $(\delta=1.0)$, and one an intermediate case with interface strength $\delta=0.5$. In reality, as also discussed by Yimsiri et al. (2004), the interface friction strength will range between $\delta=0.5$ and $\delta=1.0$, with the lower values corresponding to smooth coated or steel pipelines. However, as the difference between the intermediate interface strength case and the perfectly rough pipeline is rather small, we propose to consider eq. (10) for practical design purposes.
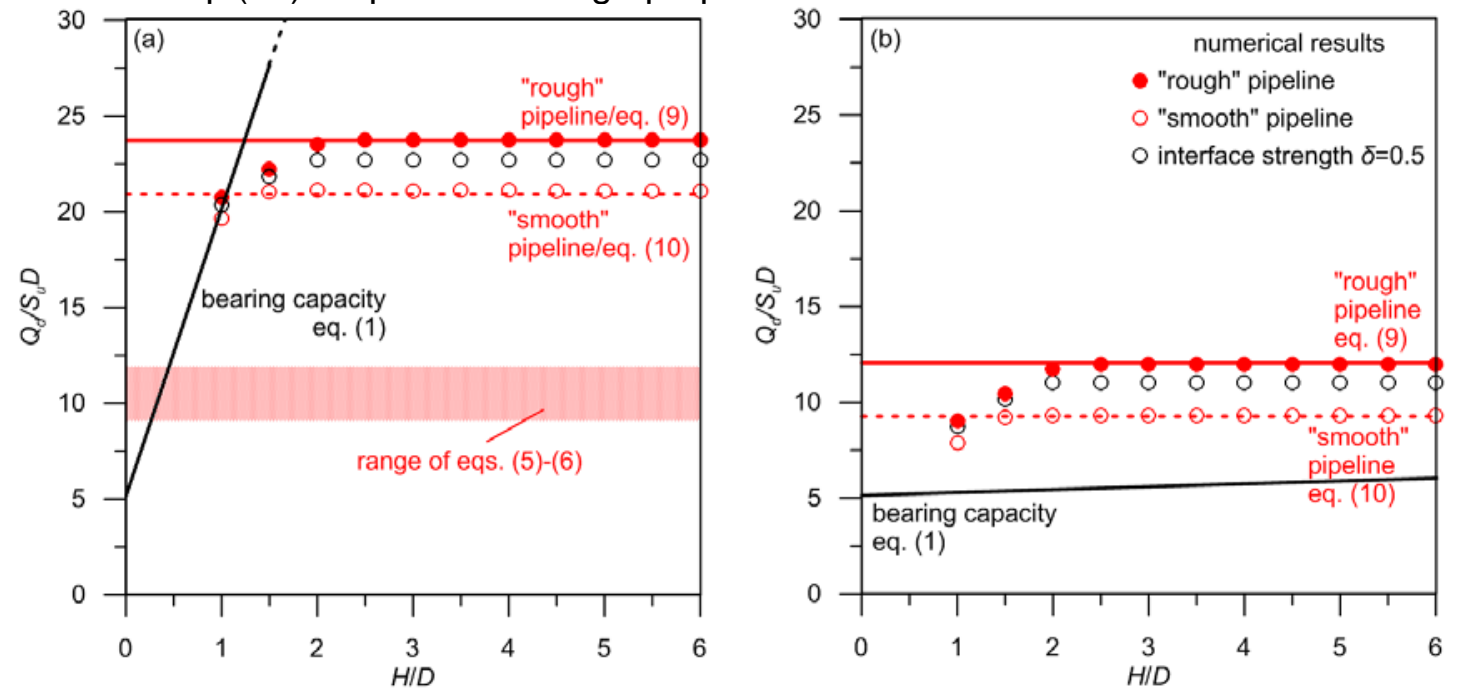

Fig. 6. Comparison of design formulas to estimate the maximum force per unit length on a pipeline embedded in cohesive soil with numerical analyses results for (a) $S_{u}=1 \mathrm{kPa} / \gamma=15 \mathrm{kN} / \mathrm{m}^{3}$ and (b) $S_{u}=100 \mathrm{kPa} / \gamma=15 \mathrm{kN} / \mathrm{m}^{3}$. 
Figure 6 shows that eq. (9-10) match the numerical results for embedment depths $H / D>2$, where the "deep" pile failure mode prevails. As expected, the force developing on the pipeline in this case is independent of the pipeline embedment, as the shear strength of the Tresca soil does not depend on the stress level. On the other hand, eq. (1) corresponding to a "shallow" footing failure mode has a clear tendency to underpredict the force on the pipeline, at least for soils with realistic undrained shear strengths.

\section{Maximum bearing force for pipelines in granular soil}

A series of parametric analyses were performed to investigate the effect of the actual embedment conditions on the maximum bearing force developing on a pipeline in granular soil. In total, we examined 11 different embedment depths ranging from $H / D=1$ to $H / D=6$ and 7 different peak friction angles ranging from $\varphi_{\text {peak }}=31^{\circ}$ to $\varphi_{\text {peak }}=44^{\circ}$ (Figure 7a). The equivalent angle concept $\varphi^{*}$ was adopted in the limit analyses, calculated according to the Davis equation mentioned in section 2.2, together with a Mohr-Coulomb failure criterion and an associative flow rule for the sand. As per section 2.2, the dilation angle to be used together with eq. (7-8) was estimated according to Bolton (1986) as $\psi=\left(\varphi_{\text {peak }}-\varphi_{\text {crit }}\right) / 0.8$. Moreover, the critical state friction angle was considered equal to $\varphi_{\text {crit }}=31^{\circ}$, from the direct shear results of Trautmann et al. (1985) in dry Cornell filter sand. The interface friction angle $\delta$ between the pipeline and the sand was taken again equal to $\delta=0.5 \varphi^{*}$.
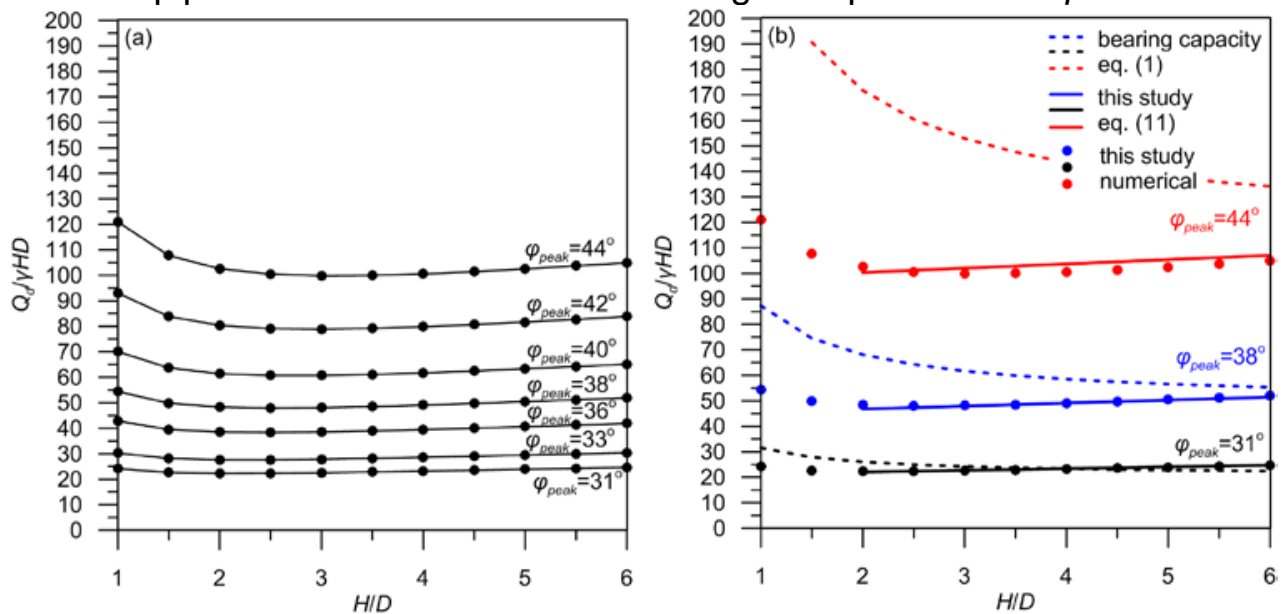

Fig. 7. (a) Maximum normalized bearing force from the parametric numerical analyses for different peak friction angles, and (b) Comparison of the curve-fitting expression (eq. 11) proposed in this study for pipelines in granular soil with the predictions of the bearing capacity eq. (1) and the numerical results, for three characteristic peak friction angles.

Focusing on embedment ratios of practical interest, which are $H / D>2$, we can use the results depicted in Figure 6a to derive the following curve-fitting expression for the estimation of the normalized bearing force per unit length of the pipeline $Q_{d} / \gamma H D$, as a function of the peak friction angle $\varphi_{\text {peak }}$ and of the embedment ratio $H / D$ :

(11) $\left(Q_{d} / \gamma H D\right)=\left(2.745 \cdot \tan \varphi_{p e c k}-0.968\right)(H / D)+1.621 \cdot e^{4.237 \cdot \tan \varphi_{\text {pest }}}$ for $H / D<2$

The normalized bearing force estimated via eq. (11) is compared against the corresponding values resulting from the bearing capacity eq. (1) in Figure $7 b$, for three peak friction angles covering the full range from loose to dense sands. Both formulas result in very similar predictions for a loose sand $\left(\varphi_{\text {peak }}=31^{\circ}\right)$. However, the bearing capacity eq. (1) which accounts for the "shallow" failure mode, overestimates the force on the pipeline by up to $70 \%$ with increasing sand density and decreasing pipeline embedment. 


\section{Trench effects on the ultimate force developing on the pipeline}

Buried pipelines are commonly installed in trenches, and subsequently backfilled with loose sand. According to the ASCE-ALA (2005) guidelines, in areas where differential ground movements are expected, the dimensions of the trench must be adequate so as the pipeline response will not be affected by the properties of the possibly much stiffer surrounding soil. Alternatively, the spring forces should be estimated while considering the native soil properties, an approach that could lead to over-design of the pipeline. However, the referenced design guidelines do not propose a method to verify that the pipeline movement will not be affected by the native soil; it is up to the pipeline designer to estimate the geometry of the developing failure surface, and the necessary trench dimensions to contain it within the sand backfill.
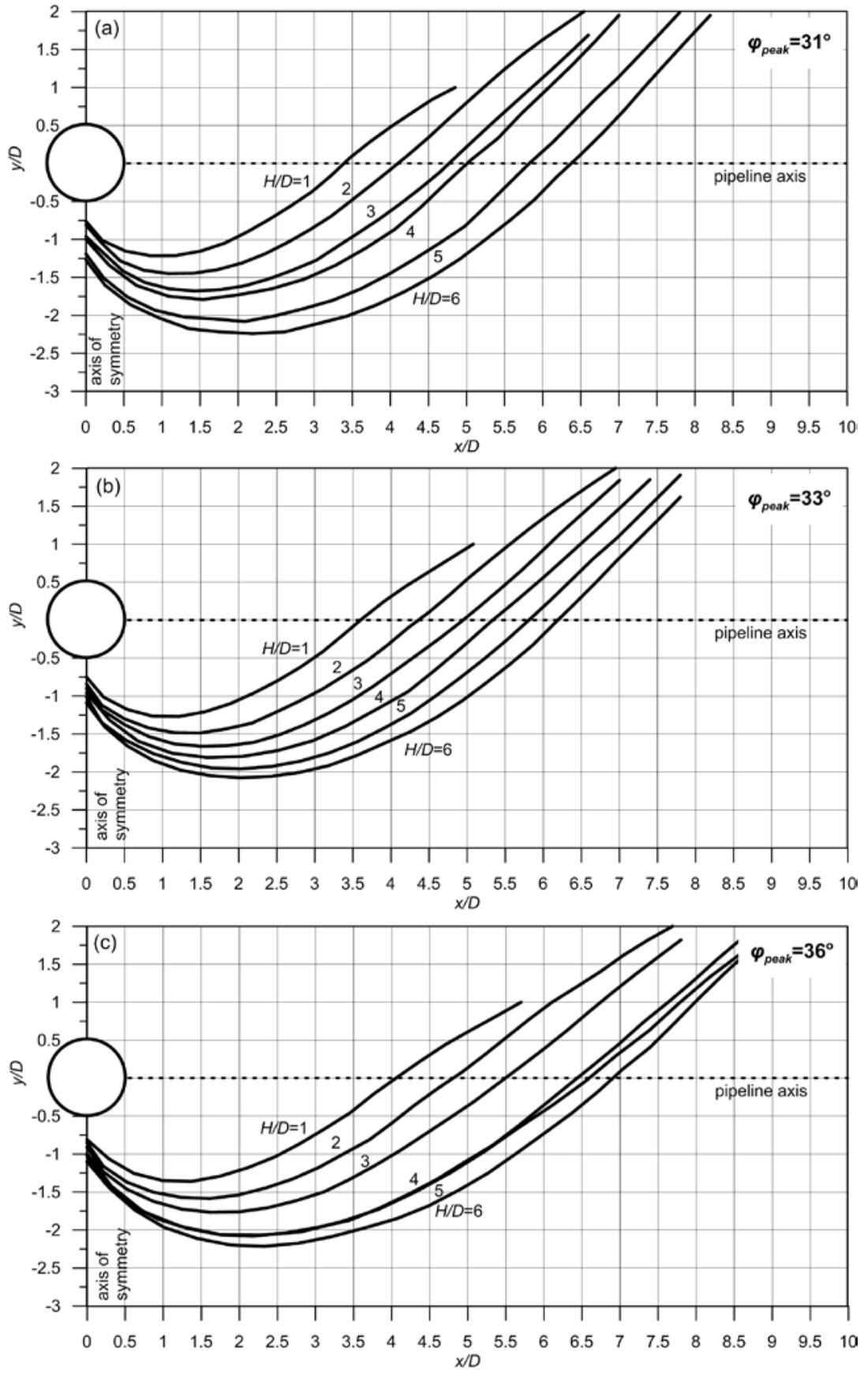

Fig. 8. Minimum trench outline to ensure failure within the sand backfill for peak friction angles (a) $\varphi_{\text {peak }}=31^{\circ}$, (b) $\varphi_{\text {peak }}=33^{\circ}$, and (c) $\varphi_{\text {peak }}=36^{\circ}$. The origin of the coordinate system $(x / D=0, y / D=0)$ refers to the final state, after pipeline movement. 
In this study, we attempt to estimate the minimum required trench dimensions so that the backfill-pipeline interaction is not affected by the native soil. The parametric analyses include different pipeline embedment depths and backfill sand friction angles. We focus on loose-to-medium sand backfills, as compaction of the backfill in practice should be restricted to a minimum in areas where differential movements are expected, to avoid the development of excessive forces on the pipeline (see Figure 7). The practical outcome of these parametric analyses is the minimum required trench shape to contain the failure surface presented in Figure 8, with the latter being a function of the pipeline diameter, embedment ratio and backfill friction angle. Note that for embedment ratios $H / D>2$, the trench slopes depicted in Figure 8 should be projected to the ground surface, following the same inclination.

The geometry of the failure surfaces in Figure 8 clearly depends on the embedment conditions of the pipeline and the passive prism is quite extensive, implying that the necessary cost related to trench works to avoid any interaction of the pipeline with the surrounding soil can be significant. Good engineering practice suggests that an optimization procedure should be followed to compensate between the increased cost of excavation works, and the possible cost of mitigation measures (e.g. use of a higher grade of steel for the pipeline) due to the increased force on the pipeline during relative offset. For example, Figure 9 presents two different analysis scenarios of a pipeline laid inside a trench and backfilled with loose sand $\left(\varphi_{\text {peak }}=31^{\circ}\right.$, $\gamma=14.8 \mathrm{kN} / \mathrm{m}^{3}$ ), at a depth $H / D=3$.

The trench in Figure $9 \mathrm{a}$ is dimensioned according to Figure 7 , while the trench in Figure $9 \mathrm{~b}$ is formed with slope inclination 3:2, common for a pipeline constructed in stiff native soil. In both cases the surrounding native soil is simulated as rigid, while the interface between the backfill sand and the soil is modeled as rough $(\delta=1.0)$. As expected, containing the shear failure by the trench walls increases the force on the pipeline, with the dimensionless force $Q_{b} / \gamma H D$ increasing from $Q_{b} / \gamma H D=22.74$ in the case of Figure $9 \mathrm{a}$ to $Q_{b} / y H D=31.19$ in the case of Figure $9 \mathrm{~b}$. Whether this difference justifies the necessary excavation costs to built an enlarged trench in the area of expected differential offset is a project-specific question, and similar numerical analyses could prove useful for the optimization of the pipeline-trench system.
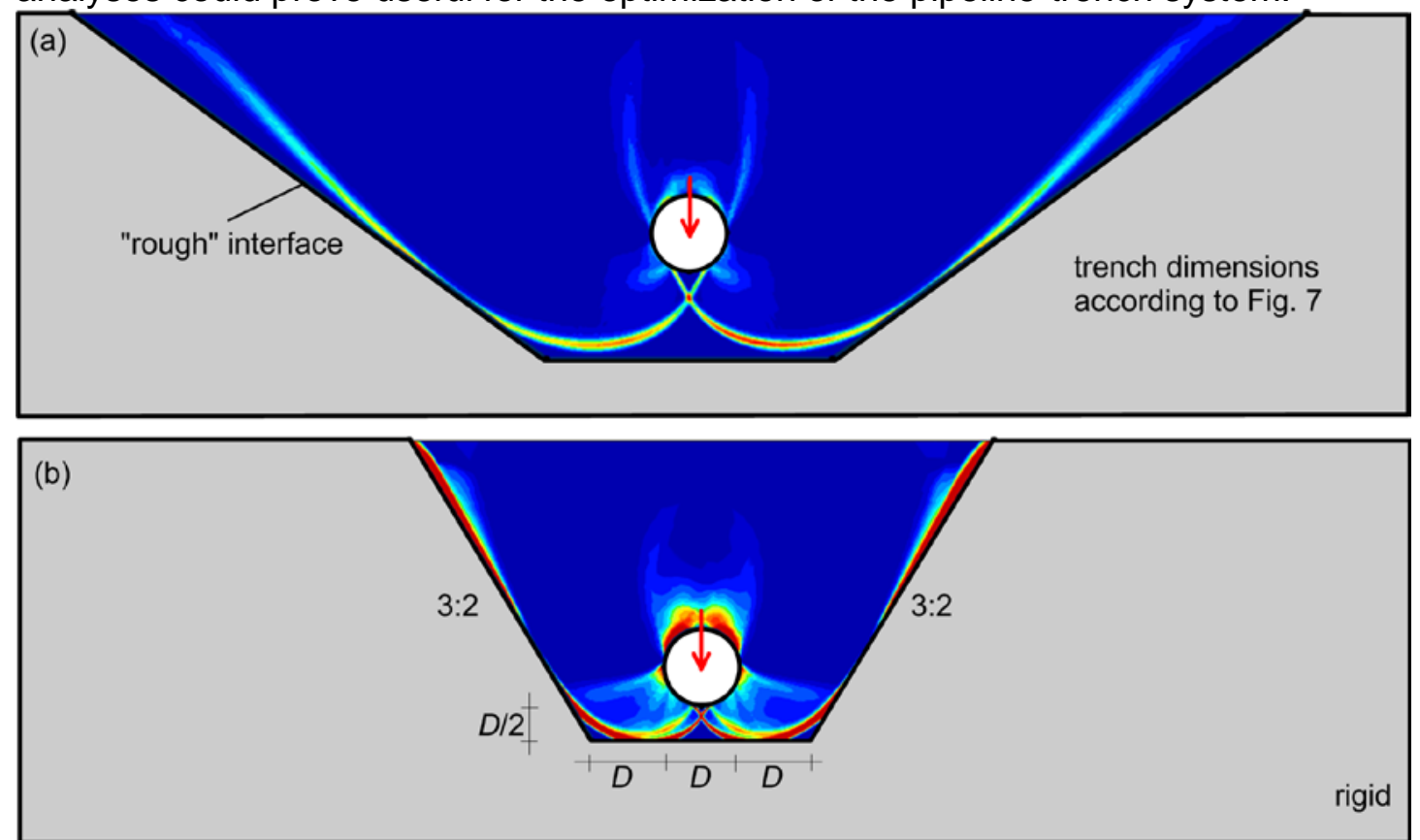

Fig. 9. Failure surface defined via maximum shear strain $\gamma_{\max }=\left|\varepsilon_{1}-\varepsilon_{3}\right|$ contours for a pipeline embedded in a loose sand-backfilled trench with (a) dimensions estimated according to Figure 8 and (b) typical dimensions and slope inclination 3:2. 


\section{Summary and conclusions}

A study on buried pipeline-soil interaction effects was presented, based on the concept that the problem of vertical downwards pipeline offset in uniform soil is equivalent to the problem of lateral loading of a long circular pile up to its collapse load. Simple, easy-to-use expressions are proposed for the estimation of the maximum force on the pipeline while accounting for the effect of the embedment conditions. These expressions can be used to calculate the vertical downwards elastoplastic spring yield force of beam-spring models, which are employed in practice to calculate pipeline strains in imposed displacement problems. Comparison against the shallow footing bearing capacity equations used in buried pipeline design practice (ASCE-ALA, 2005, O' Rourke and Liu, 2012) reveals that these equations can significantly underestimate (or in some cases overestimate) the maximum force on the pipeline, depending on the soil conditions and the geometry of the problem.

Further to the above, a quantitative method is proposed for estimating the necessary trench dimensions to prevent interaction with the possibly much stiffer native soil, for the common case of pipelines laid in sand-backfilled trenches. For cases where the cost of enlarging the trench is comparable to the cost of adopting measures to increase the strength of the pipeline, similar analyses can be used to optimize the trench dimensions with respect to the maximum force that can develop on the pipeline before reaching failure.

\section{References}

American Lifelines Alliance ALA. 2005. Guidelines for the design of buried steel pipes. New York: ASCE.

Bolton, M.D. 1986. The strength and dilatancy of sands. Géotechnique, 36(1): 65-78.

Chen, W.F. and Han, D.J. 2007. Plasticity for Structural Engineers. J. Ross Publishing.

Cocchetti, G., di Prisco, C., Galli, A., and Nova, R. 2009. Soil-pipeline interaction along unstable slopes: a coupled three-dimensional approach. Part 1: Theoretical formulation. Canadian Geotechnical Journal, 46(11): 1289-1304.

Davis, E.H. 1968. Theories of plasticity and the failure of soil masses. In Soil mechanics: selected topics. Edited by I. K. Lee. Butterworth, London. pp. 341-380.

Jefferies, M.G. 1993. Nor-Sand: A simple critical state model for sand. Geotechnique. 43(1): 91-103.

Jung, J., O' Rourke, T. and Olson, N. 2013a. Lateral soil-pipeline interaction in dry and partially saturated sand. J. Geotech. Geoenviron. Eng. 139(12): 2028-2036.

Jung, J. K., O'Rourke, T., \& Olson, N. A. 2013b. Uplift Soil-Pipe Interaction in Granular Soil. Canadian Geotechnical Journal. 50(7): 744-753.

Karamitros, D.K., Bouckovalas, G.D. and Kouretzis, G.P. 2007. Stress analysis of buried steel pipelines at strike-slip fault crossings. Soil Dynamics and Earthquake Engineeering. 27(3): 200-211.

Karamitros, D.K., Bouckovalas, G.D., Kouretzis, G.P. and Gkesouli V. 2011. An analytical method for strength verification of buried steel pipelines at normal fault crossings. Soil Dynamics and Earthquake Engineering. 31(11): 1452-1464. 
Kouretzis, G.P., Sheng, D. and Sloan S.W. 2013. Sand-pipeline-trench lateral interaction effects for shallow buried pipelines. Computers and Geotechnics. 54: 5359.

Krabbenhøft, K., Lyamin, A. V., Hjiaj, M. and Sloan, S. W. 2005. A new discontinuous upper bound limit analysis formulation. International Journal for Numerical Methods in Engineering. 63(7): 1069-1088.

Krabbenhøft, K., Lyamin, A. V. and Sloan, S. W. 2007. Formulation and solution of some plasticity problems as conic programs. International Journal of Solids and Structures. 44(5): 1533-1549.

Lyamin, A. V. and Sloan, S. W. 2002a. Lower bound limit analysis using nonlinear programming. International Journal for Numerical Methods in Engineering. 55(5): 573-611.

Lyamin, A. V. and Sloan S. W. 2002b. Upper bound limit analysis using linear finite elements and nonlinear programming. International Journal for Numerical and Analytical Methods in Geomechanics. 26(2): 181-216.

Martin, C.M. and White, D.J. 2012. Limit analysis of the undrained capacity of offshore pipelines. Geotechnique. 62(9): 847-863.

Merifield, R.S., Sloan, S.W. and Yu, H.S. 2001. Stability of plate anchors in undrained clay. Geotechnique. 51(2): 141-153.

Merifield, R.S. and Sloan, S.W. 2006. The ultimate pullout capacity of anchors in frictional soils. Canadian Geotechnical Journal. 43(8): 852-868.

O' Rourke, M.J. and Liu, X. 2012. Seismic design of buried and offshore pipelines. MCEER Monograph MCEER-12-MN04.

Olson, N. A. 2009. Soil performance for large scale soil-pipeline tests. PhD thesis, Cornell University, Ithaca, NY.

Optum G2. 2014. Optum Computational Engineering, www.optumce.com.

Oswell, J. M. 2011. Pipelines in permafrost: geotechnical issues and lessons. Canadian Geotechnical Journal. 48(9): 1412-1431.

Paulin, M.J., Philips, R., Clark, J.I., Trigg, A. and Konuk, I. 1998. A full-scale investigation into pipeline/soil interaction. In Proceedings of the $2^{\text {nd }}$ International Pipeline Conference, Calgary, Canada.

di Prisco, C. and Galli, A. 2006. Soil-pipe interaction under monotonic and cyclic loads: experimental and numerical modelling. In Proceedings of the $1^{\text {st }}$ EuroMediterranean Symposium on "Advances in Geomaterials and Structures", Hammamet, Tunisia.

Rajeev, P. and Kodikara, J. 2011. Numerical analysis of an experimental pipe buried in swelling soil. Computers and Geotechnics. 38(7): 897-904.

Randolph, M.F. and Houlsby, G.T. 1984. The limiting pressure on a circular pile loaded laterally in cohesive soil. Geotechnique. 34(4): 613-623. 
Robert, D. and Soga, K. 2010. Soil-pipeline interaction in unsaturated sands. In Mechanics of unsaturated geomaterials. Edited by L. Laloui. ISTE Ltd. and John Wiley \& Sons, Inc. pp. 303-325.

Rojhani, M., Moradi, M., Galandarzadeh, A., and Takada, S. 2012. Centrifuge modeling of buried continuous pipelines subjected to reverse faulting. Canadian Geotechnical Journal. 49(6): 659-670.

Shi, J., Wang, Y., and Ng, C. W. 2013. Buried pipeline responses to ground displacements induced by adjacent static pipe bursting. Canadian Geotechnical Journal. 50(5): 481-492.

Sloan, S. W. 1988. Lower bound limit analysis using finite elements and linear programming. International Journal for Numerical and Analytical Methods in Geomechanics. 12(1): 61-67.

Sloan, S. W. 1989. Upper bound limit analysis using finite elements and linear programming. International Journal of Numerical and Analytical Methods in Geomechanics. 13(3): 263-282.

Sloan, S. W. 2013. Geotechnical stability analysis. Géotechnique. 63(7): 531-572.

Sloan, S. W. and Kleeman, P. W. 1995. Upper bound limit analysis using discontinuous velocity fields. Computer Methods in Applied Mechanics and Engineering. 127(1-4): 293-314.

Trautmann, C.H. and O' Rourke, T.D. 1985. Lateral force-displacement response of buried pipe. J. Geotech. Engng ASCE. 111(9): 1068-1084.

Trautmann, C.H., O' Rourke, T.D. and Kulhawy, F.D. 1985. Uplift force-displacement response of buried pipe. J. Geotech. Engng ASCE. 111(9): 1061-1076.

Turner, J.E. 2004. Lateral force-displacement behaviour of pipes in partially saturated sand. MS thesis, Cornell University, Ithaca, NY.

Yimsiri, S., Soga, K. Yoshizaki, K. Dasari, G. and O' Rourke, T.D. 2004. Lateral and upward soil-pipeline interactions in sand for deep embedment conditions. J. Geotech. Geoenviron. Engng ASCE. 130(8): 830-842.

Wang, Y., Shi, J., and Ng, C. W. 2011. Numerical modelling of tunnelling effect on buried pipelines. Canadian Geotechnical Journal. 48(7): 1125-1137.

Wols, B.A. and van Thienen, P. 2014. Modelling the effect of climate change induced soil settling on drinking water distribution pipes. Computers and Geotechnics. 55: 240-247. 